\title{
Evaluation of Rutting Parameters of Asphalt Binder Based on Rheological Test
}

\author{
Chao Wang and Jinxi Zhang
}

\begin{abstract}
This study evaluated the rutting potential of two types of asphalt binders namely the neat binder and SBS modified binder by respectively using the oscillation-based parameter $G * / \sin \delta$ and the oscillation-based nonrecoverable stiffness parameter $G * /(1-(1 / \tan \delta \sin \delta))$ as well as the MSCR-based nonrecoverable compliance Jnr. Both original and short-term aged asphalt binders were tested in this evaluation. Test results indicates that the parameter $G * /(1-(1 / \tan \delta \sin \delta))$ exhibits a stronger correlation with the parameter Nonrecoverable Compliance Jnr than that of parameter G*/sin $\delta$ which means the parameter $G * /(1-(1 / \tan \delta \sin \delta))$ can capture well the nonrecoverable response and should be a better choice than $G * / \sin \delta$ when using the oscillation test to evaluate the rutting potential of asphalt binder.
\end{abstract}

Index Terms-Asphalt binder, rheological test, rutting parameters, correlation analysis.

\section{INTRODUCTION}

In recent decades asphalt pavements have been developed rapidly for highway construction in China. However, following the fast building speed, the pavements quality also gradually arouses more and more attentions on the durable performance of the asphalt materials. Permanent deformation, which is usually accumulated in summer high temperature condition and described as rutting damage, is crucial for pavements service level especially for high-speed expressway. As one of the main raw materials, asphalt binder rutting potential plays a dominant role in the deformation feature of asphalt mixture and the eventual pavements rutting performance. So measuring and evaluating the material properties based on the performance-related test is becoming significantly essential to select proper and effective asphalt binder for pavements design and construction.

In 1993, the American Strategic Highway Research Program (SHRP) introduced the rheological approach to characterize the performance potential of asphalt binder by using Dynamic Shear Rheometer (DSR) to determine the complex shear modulus $\mathrm{G}^{*}$ and the phase angle $\delta$ in oscillation test , and then $\mathrm{G}^{*} / \sin \delta$ can be calculated and regarded as the rutting parameter of asphalt binder [1]. However, the SHRP

\footnotetext{
Manuscript received March 14, 2013; revised July 5, 2013.This work was supported in part by the Natural Science Foundation of Beijing Government under Grant 8100001 and Science Technology Program of Hebei Province Transportation Administration under Grant Y-2010154.

Chao Wang is with the Transportation Research Center, Beijing University of Technology, Beijing, 100124, China (e-mail: chaowang1120@sina.com).

Jinxi Zhang is with the Transportation Research Center, Beijing University of Technology, Beijing, 100124, China (e-mail: zhangjinxi@bjut.edu.cn).
}

research work mainly focused on the unmodified neat asphalt binders so that applicability of parameter $\mathrm{G}^{*} / \sin \delta$ to modified binders has always been questioned due to the fact that strain recovery ability (delayed elastic) of the modified binders is much excellent than the neat binders. In 2001, based on the same oscillation test approach as SHRP rutting parameter $G^{*}$ $/ \sin \delta$, Shenoy began to develop a parameter $\mathrm{G}^{*} /(1-(1 / \tan \delta \sin \delta))$ that essentially captures the nonrecoverable response of asphalt binders, which makes the oscillation test approach more suitable to modified binders [2].

In 2001, NCHRP 9-10 employed a new test method namely Repeated Creep Recovery (RCR) test to determine the nonrecoverable compliance of asphalt binders for the high-temperature specification as it relates to the binder contributions in the field pavements permanent deformation [3]. And recently in 2010, D'Angelo extended the NCHRP 9-10 binder creep and recovery characterization approach and suggested a further refined simple high-temperature binder specification through the use of Multiple Stress Creep Recovery (MSCR) test, which introduces stress sensitivity and a recoverable strain response along with the nonrecoverable response [4]. And the nonrecoverable compliance $\mathrm{J}_{\mathrm{nr}}$ determined from MSCR test has been developed as an alternative to SHRP rutting parameter $\mathrm{G}^{*} / \sin$ $\delta$.

This paper firstly evaluated the rutting potential of two types of asphalt binders namely the neat binder and SBS modified binder by respectively using the SHRP oscillation-based parameter $\mathrm{G}^{*} / \sin \delta$, the oscillation-based nonrecoverable stiffness parameter $G^{*} /(1-(1 / \tan \delta \sin \delta))$ as well as the MSCR-based nonrecoverable compliance $\mathrm{J}_{\mathrm{nr}}$, and then focused on the correlation identification between the three different parameters. Besides, both original and short-term aged asphalt binders were tested in this evaluation.

\section{EXPERIMENTAL INVESTIGATION}

\section{A. Materials}

TABLE I: PHYSICS PROPERTIES OF ASPHALT BINDERS

\begin{tabular}{ccc}
\multicolumn{2}{c}{ TABLE I: PHYSICS PROPERTIES OF ASPHALT BINDERS } \\
\hline \hline Designation & Neat Binder & $\begin{array}{c}\text { SBS Modified } \\
\text { binder }\end{array}$ \\
\hline $\begin{array}{c}\text { Penetration at } \\
25^{\circ} \mathrm{C}, 100 \mathrm{~g}, 5 \mathrm{~s}\end{array}$ & 70.67 & 61 \\
$(0.1 \mathrm{~mm})$ & & \\
Softening point $\left({ }^{\circ} \mathrm{C}\right)$ & 43.40 & 61.25 \\
Ductility at $15^{\circ} \mathrm{C}(\mathrm{mm})$ & 136.24 & 93.64 \\
Ductility at $5^{\circ} \mathrm{C}(\mathrm{mm})$ & - & 22.73 \\
\hline \hline
\end{tabular}


Two types of asphalt binders were used in this study, namely the neat asphalt and the SBS polymer-modified asphalt. The traditional physics properties of binders which consists Penetration at $25^{\circ} \mathrm{C}$, Softening point as well as Ductility at $15^{\circ} \mathrm{C}$ and $5^{\circ} \mathrm{C}$ are showed in Table I.

\section{B. Test Methods}

There are totally three binder experimental methods employed to determine the various binder characteristics.

Rolling Thin-Film Oven Test. The asphalt binder aged in the process of pavements construction is known as short-term ageing. As shown in Fig. 1, Rolling Thin-Film Oven was employed to simulate the short-term ageing of both neat and SBS modified binders in accordance with the short-term ageing procedure AASHTO T240 [5], then the test binders residues, which can be defined as RTFOT binder, were prepared to the followed measurement of rheological properties, which would provide a comparison with that of the ordinary unaged binders.

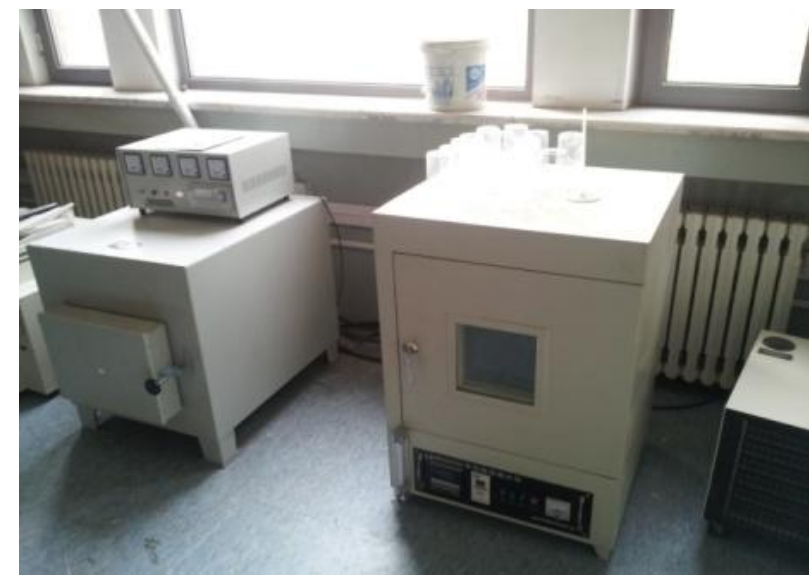

Fig. 1. Rolling thin-film oven.

Oscillation Test. The high temperature rheological properties of each binder were measured using an oscillation test conducted on the dynamic shear rheometer (DSR) according to AASHTO T315-06 [1]. As shown in Fig. 2, the DSR equipment has been widely accepted by highway agencies in U.S. and some research institutes in China to identify the rheological behaviors of asphalt binders in specifications. The DSR measures the stress strain response of viscoelastic materials and is ideally suited to evaluate asphalt binders. As one of six standard tests provided by DSR, oscillation test is used to measure the complex shear modulus $\mathrm{G}^{*}$ and the phase angle $\delta$ under a oscillatory sinusoidal shear condition, thus the PG high temperature grade of asphalt binders can be determined by calculating the SHRP rutting potential parameter $\mathrm{G}^{*} / \sin \delta$ according to AASHTO M320 [6].

Multiple Stress Creep Recovery Test. The MSCR test uses the well-established creep and recovery test concept to evaluate the binder's potential for permanent deformation, and the equipment of the new test is also focused on the existing DSR. Different from the oscillation test used in the existing PG specification, the creep/creep recovery standard test on the DSR is employed. Firstly a one-second creep load was applied to the asphalt binder specimen, then the load was removed and the specimen was allowed to recovery for 9 seconds. The test was started with the application of a low stress level of $0.1 \mathrm{kPa}$ for 10 creep/recovery cycles and then increased to $3.2 \mathrm{kPa}$ for additional repeated 10 cycles with no time lags between the two stress levels. The total time of the test is 200 seconds and the new evaluation parameters are average Recovery Percent R and Nonrecoverable Compliance $\mathrm{J}_{\mathrm{nr}}$. Besides, the test temperature is the same as the binder high temperature grade determined from the oscillation test.

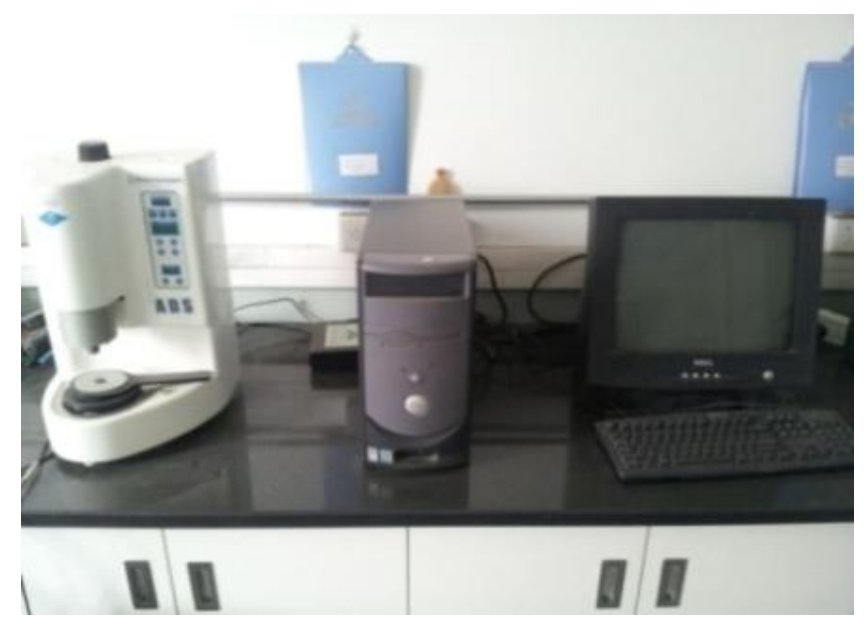

Fig. 2. Dynamic Shear Rheometer (DSR).

Fig. 3 shows the typical time-strain data from MSCR test and the determination of $\mathrm{R}$ and $\mathrm{J}_{\mathrm{nr}}$ for asphalt binders. Calculating the $\mathrm{R}$ for every cycle at one stress level, the average values from the 10 cycles can be defined as $R_{0.1}$ and $R_{3.2}$, respectively. The same calculating method can be used in the determination of $\mathrm{J}_{\mathrm{nr} 0.1}$ and $\mathrm{J}_{\mathrm{nr} 3.2}$.

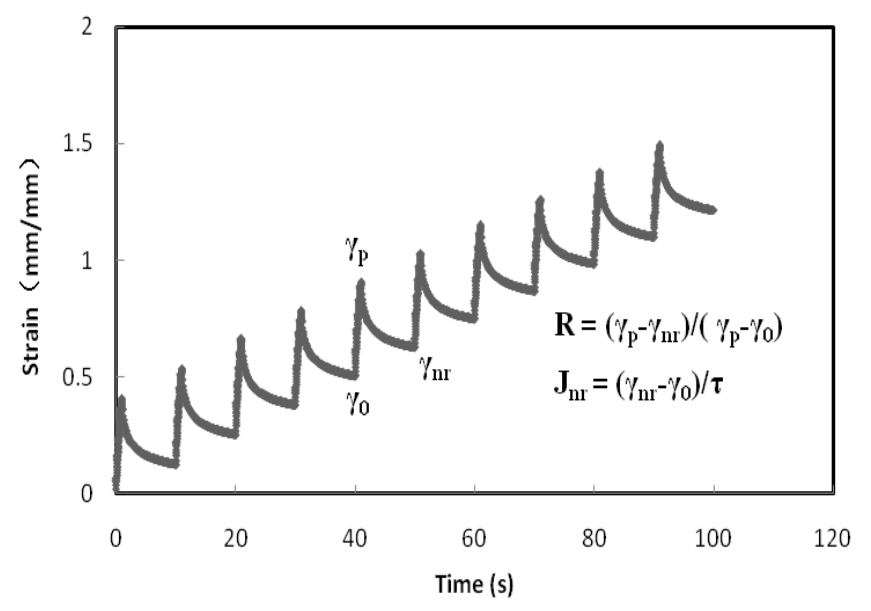

Fig. 3. Plot of the time-strain data from MSCR test.

\section{RESUlTS AND DISCUSSION}

\section{A. Oscillation-based Rutting Parameters}

In the existing asphalt binder specification AASHTO $\mathrm{M} 320$, the parameter $\mathrm{G}^{*} / \sin \delta$ calculated by the $\mathrm{G}^{*}$ and $\delta$ is not only a rutting potential indicator, but also determinate the high temperature performance grade of binders, which are required as the $\mathrm{G}^{*} / \sin \delta>1.0 \mathrm{kPa}$ for original unaged binder and $\mathrm{G}^{*} / \sin \delta>2.2 \mathrm{kPa}$ for RTFOT binder. The Oscillation test results of neat binder and SBS Modified Binder are shown in Table II and Table III , respectively. 
TABLE II: OSCILLATION TEST RESULTS OF NEAT BINDER

\begin{tabular}{ccccccc}
\hline \hline & \multicolumn{3}{c}{ Original Binder } & \multicolumn{2}{c}{$\mathrm{RTFOT}$ binder } \\
\cline { 2 - 6 } Temperature $\left({ }^{\circ} \mathrm{C}\right)$ & $\delta\left(^{\circ}\right)$ & $\mathrm{G}^{*}(\mathrm{kPa})$ & $\mathrm{G}^{*} / \sin \delta(\mathrm{kPa})$ & $\delta\left(^{\circ}\right)$ & $\mathrm{G}^{*}(\mathrm{kPa})$ & $\mathrm{G}^{*} / \mathrm{sin}^{\circ}(\mathrm{kPa})$ \\
\hline 52 & 85.87 & 4.76 & 4.68 & 73.72 & 25.47 & 26.54 \\
58 & 86.18 & 2.10 & 2.11 & 77.44 & 10.49 & 10.75 \\
64 & 87.02 & 0.81 & 0.81 & 79.85 & 4.62 & 4.69 \\
\hline \hline
\end{tabular}

TABLE III: OSCILLATION TEST RESULTS OF SBS MODIFIED BINDER

\begin{tabular}{ccccccc}
\hline & \multicolumn{3}{c}{ Original Binder } & \multicolumn{2}{c}{ RTFOT binder } \\
\cline { 2 - 7 } Temperature $\left({ }^{\circ} \mathrm{C}\right)$ & $\delta\left(^{\circ}\right)$ & $\mathrm{G}^{*}(\mathrm{kPa})$ & $\mathrm{G}^{*} / \sin \delta(\mathrm{kPa})$ & $\delta\left(^{\circ}\right)$ & $\mathrm{G}^{*}(\mathrm{kPa})$ & $\mathrm{G}^{*} / \sin \delta(\mathrm{kPa})$ \\
\hline 70 & 67.06 & 2.41 & 2.62 & 69.95 & 3.38 & 3.60 \\
76 & 67.72 & 1.43 & 1.54 & 71.11 & 1.87 & 1.97 \\
82 & 67.01 & 0.88 & 0.96 & 71.12 & 1.04 & 1.10 \\
\hline \hline
\end{tabular}

TABLE IV: OSCILLATION-BASED RUTTING PARAMETERS

\begin{tabular}{ccccc}
\hline \hline \multirow{2}{*}{ Rutting Parameters $(\mathrm{kPa})$} & \multicolumn{2}{c}{ Near Binder } & \multicolumn{2}{c}{ SBS Modified Binder } \\
\cline { 2 - 5 } & Original Binder & RTFOT Binder & Original Binder & RTFOT Binder \\
\hline $\mathrm{G} * / \sin \delta$ & 2.11 & 10.75 & 1.54 & 3.60 \\
$\mathrm{G}^{*} /(1-(1 / \tan \delta \sin \delta))$ & 1.72 & 6.67 & 1.74 & 5.53 \\
\hline \hline
\end{tabular}

TABLE V: MSCR-BASED RUTTING PARAMETERS

\begin{tabular}{ccccc}
\hline \hline Nonrecoverable & \multicolumn{2}{c}{ Near Binder } & \multicolumn{2}{c}{ SBS Modified Binder } \\
\cline { 2 - 5 } Compliance $\mathrm{J}_{\mathrm{nr}}(1 / \mathrm{kPa})$ & Original Binder & RTFOT Binder & Original Binder & RTFOT Binder \\
\hline $\mathrm{J}_{\mathrm{nr} 0.1}$ & 4.39 & 0.57 & 1.21 & 0.73 \\
$\mathrm{~J}_{\mathrm{nr} 3.2}$ & 4.77 & 0.64 & 4.73 & 1.22 \\
\hline \hline
\end{tabular}

From the Table II, it was observed that the high-temperature grade of neat binder should be $58^{\circ} \mathrm{C}$ and its corresponding RTFOT binder grade remained the same according to the $\mathrm{G}^{*} / \sin \delta$ requirement mentioned above. For SBS modified binder shown in Table III, the high-temperature grade was identified at $76{ }^{\circ} \mathrm{C}$ whereas its corresponding RTFOT binder should have to reduce to $70^{\circ} \mathrm{C}$ to meet the specification requirement. Additionally, the oscillation-based nonrecoverable stiffness parameter $\mathrm{G}^{*} /(1-(1 / \tan \delta \sin \delta))$ of each binder were also calculated at their own high-temperature grade and are provided together with the corresponding parameter $\mathrm{G}^{*} / \sin \delta$ in Table IV.

\section{B. MSCR-Based Rutting Parameter}

Based on the binder high temperature grade determined in oscillation test, all binder specimens were then measured in MSCR test for the rutting parameter Nonrecoverable Compliance $\mathrm{J}_{\mathrm{nr}}$ at their own grade temperature. The binders $\mathrm{J}_{\mathrm{nr}}$ calculation results under two stress levels are illustrated in Table V.

\section{Correlation Identification between Different Binder Rutting Parameters}

Compared with the SHRP oscillation-based rutting parameter $G^{*} / \sin \delta$, the parameter $G^{*} /(1-(1 / \tan \delta \sin \delta))$ which was determined from the same oscillation test and derived theoretically based on viscoelastic characteristic analysis, is more reasonable to represent the delayed elasticity as well as the nonrecoverable strain response of modified asphalt binders. Meanwhile, based on new loading approach of creep recovery and the calculation of Nonrecoverable Compliance $\mathrm{J}_{\mathrm{nr}}$, the MSCR test entirely can obviously capture the binder nonrecoverable response, which is always related to the permanent deformation.

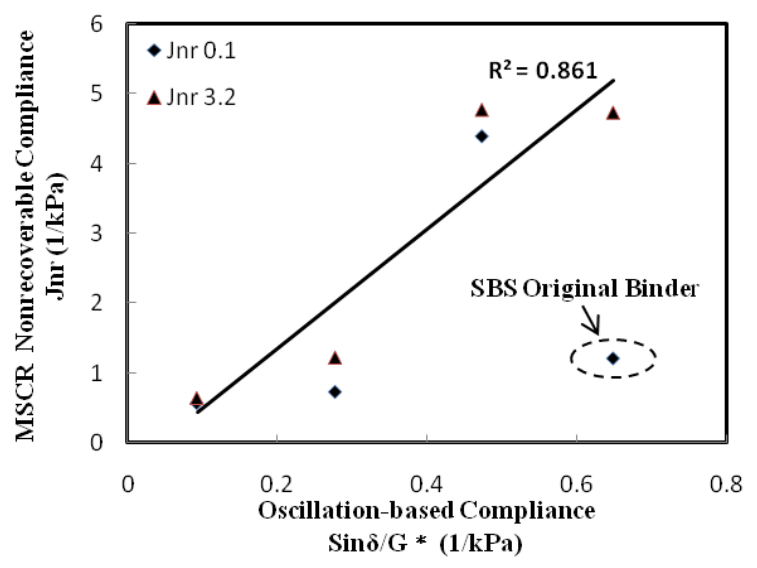

Fig. 4. Relationship between Oscillation-based $\mathrm{G} * / \sin \delta$ and MSCR-based Jnr.

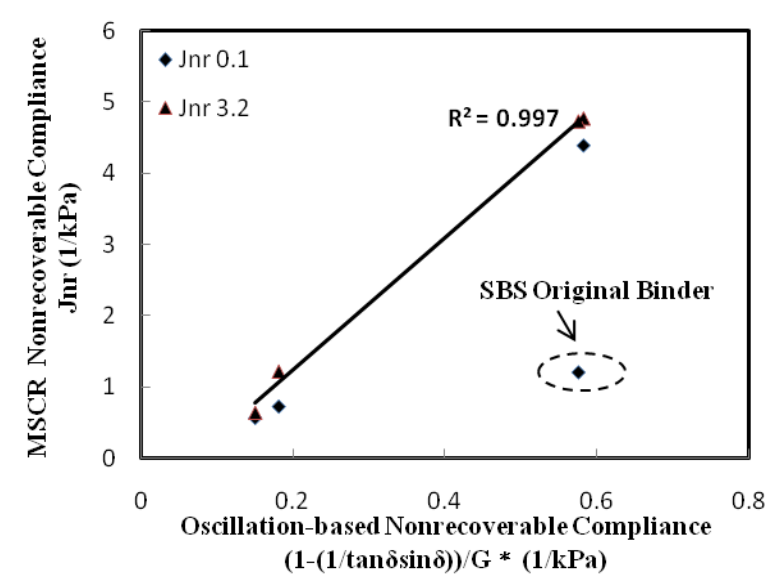

Fig. 5. Relationship between Oscillation-based $G^{*} /(1-(1 / \tan \delta \sin \delta))$ and MSCR-based Jnr.

Fig. 4 and Fig. 5 respectively show the relationships between MSCR-based parameter and the two oscillation-based parameters which are both inversed to have 
a same unite $1 / \mathrm{kPa}$ as $\mathrm{J}_{\mathrm{nr}}$. Although in each figure the two parameters are not numerically identical, they both exhibit a good relationship except for just one point resulted from a low compliance of SBS original binder under the low stress level. Especially the oscillation-based parameter $\mathrm{G}^{*} /(1-(1 / \tan \delta \sin \delta))$ is noticed to have a stronger correlation with the MSCR-based parameter Nonrecoverable Compliance $J_{n r}$ than that of the SHRP oscillation-based rutting parameter $\mathrm{G}^{*} / \sin \delta$, which indicates that using the oscillating load test under low strain amplitude level can also capture the similar nonrecoverable response equivalently to that measured in the MSCR test.

\section{SUMMARY}

This study evaluated the rutting potential of two types of asphalt binders namely the neat binder and SBS modified binder by using two kinds of test methods which consisted the Oscillation test and Multiple Stress Creep Recovery (MSCR) test. Both original and short-term aged asphalt binders were tested in this evaluation.

The two oscillation-based parameters $\mathrm{G}^{*} / \sin \delta$ and the nonrecoverable stiffness parameter $G^{*} /(1-(1 / \tan \delta \sin \delta))$ were respectively identified a relationship with the MSCR-based parameter nonrecoverable compliance $\mathrm{J}_{\mathrm{nr}}$. And the $\mathrm{G}^{*} /(1-(1 / \tan \delta \sin \delta))$ exhibits a stronger correlation with the Nonrecoverable Compliance $\mathrm{J}_{\mathrm{nr}}$ than that of parameter $\mathrm{G}^{*} / \sin$ $\delta$. This indicates that parameter $G^{*} /(1-(1 / \tan \delta \sin \delta))$ should be a better choice than $\mathrm{G}^{*} / \sin \delta$ when using oscillation test to evaluate the rutting potential of asphalt binder.

\section{REFERENCES}

[1] Standard Method of Test for Determining the Rheological Properties of Asphalt Binder Using a Dynamic Shear Rheometer (DSR), AASHTO T 315-06, 2006.

[2] N. Gibson, X. Qi, and A. Shenoy, Performance Testing for Superpave and Structural Validation, U.S. Department of Transportation, Federal Highway Administration, Report Publication No. FHWA-HRT-11-045, November, 2012.

[3] H. U. Bahia, D. I. Hanson, M. Zeng, H. Zhai, M. A. Khatri, and R. M. Anderson, Characterization of Modified Asphalt Binders in Superpave Mix Design, Transportation Research Board, National Academy Press, Washington D.C., 2001.

[4] Standard Method of Test for Multiple Stress Creep Recovery (MSCR) Test of Asphalt Binder Using a Dynamic Shear Rheometer (DSR), AASHTO TP 70-10, 2010.
[5] Standard method of test for effect of heat and air on a moving film of asphalt binder (rolling thin-film oven test), AASHTO T 240, Washington D.C., 1997.

[6] Standard Specification for Performance-Graded Asphalt Binder, AASHTO M 320-05, 2005.

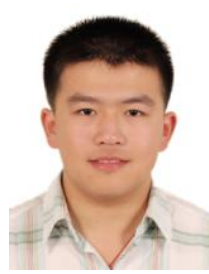

Chao Wang was born in Handan, Hebei, China on November 20, 1986 earned his bachelor degree of Civil Engineering in Chongqing Jiaotong University (CQJTU) in June 2010.

He is currently studying on the Road Engineering as a Ph.D. candidate in Transportation Research Center in Beijing University of Technology (BJUT) and his research topic mainly focuses on the asphalt pavements especially on the rutting potential of the asphalt

materials

Mr. Wang now is a student member of the Association of Asphalt Paving Technologists (AAPT) which is a leader in the advancement of asphalt paving technology in United States

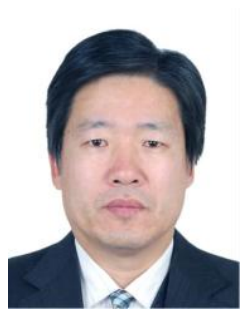

Jinxi Zhang was born in Hebei, China at March 28, 1965. He obtained the bachelor of Engineering, Highway engineering in Hebei University of Technology, Tianjin city of China in 1985; and the mater degree in Urban Road Engineering, Beijing Municipal Engineering Research Institute, Beijing city of China in 1988 . He got the doctoral degree in Civil and Environmental Engineering, Iwate University, Morioka city of Japan in 2000. His major fields of study is pavement materials including asphalt concrete and cement concrete, the performance of pavement surface and durability of traffic facilities.

$\mathrm{He}$ worked as a Road engineer in Beijing Municipal Engineering Research Institute from 1988 to 1993, and as a director and engineer in Municipal Engineering Bureau of Beijing City Government from 1994 to 1996, as an assistant professor in Iwate University from 2000 to 2002 and as an associate professor in Iwate University from 2002 to 2003. From 2003 to now, he is working as a professor in Beijing University of Technology, Beijing, China. He published two books including "Recycling Technology of Pavement Materials" (Beijing, China, Science Press, 2008) and "Road Engineering Monograph” (Beijing, China, Science Press, 2010).

Prof. Zhang is the director of Road Engineering Branch Society of China Highway and Transportation Society, the executive director of Beijing Highway Society and the member of Road and Bridge Standardization Committee of the Ministry of Housing and Urban-rural Development of the People's Republic of China. He got "Science and Technology Award of Beijing Government" in 1992 and "Science and Technology Award of China Highway and Transportation Society" in 2008. 